\title{
A historical method approach to teaching Kepler's 2nd law
}

\author{
Wladimir Lyra ${ }^{1, *}$ \\ ${ }^{1}$ New Mexico State University, USA \\ *wlyra@nmsu.edu
}

\begin{abstract}
Kepler's 2 nd law, the law of the areas, is usually taught in passing, between the 1st and the 3rd laws, to be explained "later on" as a consequence of angular momentum conservation. The 1st and 3rd laws receive the bulk of attention; the lst law because of the paradigm-shift significance in overhauling the previous circular models with epicycles of both Ptolemy and Copernicus, the 3rd because of its convenience to the standard curriculum in having a simple mathematical statement that allows for quantitative homework assignments and exams. In this work I advance a method for teaching the 2 nd law that combines the paradigm-shift significance of the lst law and the mathematical proclivity of the 3rd law. The approach is rooted in variational learning and the historical method, indeed, placed in its historical context, Kepler's 2 nd is as revolutionary as the 1st: as the 1 st law does away with the epicycle, the 2 nd law does away with the equant. This way of teaching the 2 nd law also formulates the "time=area" statement quantitatively, in the way of Kepler's equation, $M=E-e \sin E$, (relating mean anomaly $M$, eccentric anomaly $E$, and eccentricity $e$ ), where the left-hand side is time and the right-hand side is area. In doing so, it naturally paves the way to finishing the module with an active learning computational exercise, for instance, to calculate the timing and location of Mars' next opposition. This method is partially based on Kepler's original thought, and should thus best be applied to research-oriented students, such as junior and senior physics/astronomy undergraduates, or graduate students.
\end{abstract}

Keywords: Culture-Based Astronomy Education Research; Astronomy Laboratory Activities; Kepler's Laws; Historical Method; Variational Theory of Learning.

\section{Introduction}

Kepler's 2nd law is arguably the most challenging of Kepler's laws to teach. Yu et al. (2010) found that, in a sample of 112 undergraduate student interviews to gauge prior knowledge for an introductory astronomy course, the majority (54\%) declined to even guess an answer when inquired about it. This contrasts to the lst and 3rd laws, where a majority of the same students gave incomplete but correct statements about them ("orbits are not circles", and "planets orbiting closer to the Sun move faster; those orbiting farther move slower", respectively). While the sample of Yu et al. (2010) was of nonmajor freshmen, the 2 nd law remains underrated in upper division major undergraduate courses, where students' understanding of it still lingers on the qualitative, and divorced from its historical significance. In addition, Aktan and Dinçer (2014) find alternative conceptions about the 2 nd law even among pre-service science teachers. This evidences shortcomings about the way the law is traditionally explained. The 2 nd law is frequently taught (e.g.: Carroll and Ostlie, 2007; Ryden and Peterson, 2020) as a variation of the following statement:

A line drawn from the Sun to a planet sweeps out equal areas in equal time intervals.

This sentence is usually followed by diagrams showing short wide areas near the Sun and long slender areas far away. "They're equal!", says the instructor, almost like a curiosity. "Why 
is this important?", asks the inquisitive student. Planets go fast near the Sun and slow far from it, is the usual answer. It is how it is described in popular astronomy books (e.g.: de Cayeux and Brunier, 1983), in high school physics (e.g.: Kuhn, 1962; Guimaraes and Fonte Boa, 2006), and in numerous educational websites. In college, one walks the extra mile of proving from Newton's laws that Kepler's 2nd simply reflects angular momentum conservation (e.g.: Halliday and Resnick, 2020; Marion and Thornton, 2020). This is usually done by climbing down the pedestal of differential and integral calculus to the pedestrian world of Euclidian geometry and defining the strange concept of "areal velocity", which is then proved to be constant.

The historical inversion (Newton before Kepler) is rooted on a discipline approach (Rice, 1972). By grounding Kepler's 2nd law on angular momentum conservation, it draws on principled conceptual knowledge (Leinhardt, 1988), facilitating learning by structuring it around a major concept of the discipline of physics. On the other hand, teaching the material in this way has the unfortunate drawback of reducing Kepler's 2nd law to a post-factum instead of presenting it as the product of original logic, painstaking problem-solving, and what was then cutting-edge research. These, in turn, are precisely the skills that should be developed in high-ability students (Dixon et al., 2004). More importantly, this presentation fails to obviate that the 2 nd law is about quantitatively finding the planet in the orbit. In this paper I develop a model for teaching Kepler's second law using the historical method (Matthews, 1989; Coelho, 2009; Galili, 2010). The proposed teaching sequence partially recreates in the classroom the historical perspective in which Kepler discovered the law in Astronomia Nova (Kepler, 1609; Aiton, 1969; Boccaletti, 2001).

\section{The Historical Method}

The historical method (also called genetic approach) has a rich record in physics. Mach $(1895,1911)$ and Duhem (1906) argued that retracing the original line of thought in discovering the laws of nature led to a deeper understanding of the subject by the novice students. The sentiment is echoed by Schwab (1962), who defined teaching science as inquiry in its essence as "to show some of the conclusions of science in the framework of the way they arise and are tested". Modern pedagogy frames this postulate under the idea of cognitive recapitulation (Piaget, 1970; Posner et al., 1982): ontogeny recapitulates phylogeny, i.e. there is a parallel between how an individual accrues knowledge (ontogeny) and how the knowledge in the discipline itself evolved (phylogeny).

Indeed, the way by which conceptual change is brought about in learners (Posner et al., 1982) shares many similarities with the structure of paradigm shifts (Kuhn, 1962). According to Posner et al. (1982), learners have no urge to change their conception until that conception fails at problem solving. This is how scientific theories are constructed, thriving until anomalous data is introduced, that cannot be accounted for by the prevailing model. Also, the first response of learners to anomalous data is not dismissal of the old conception, but questioning of the data. This is mirrored by the healthy skepticism of the scientific community, requiring extraordinary claims to be backed by extraordinary evidence. Finally, once the quality of the new data is established, learners arrive at a conceptual change when presented with a new theory that accounts for both the old and the new data. This is epitomized by the correspondence principle (Bohr, 1920), which requires a new theory to explain all the phenomena for which a preceding theory was valid. Visibly, the historical method retraces the development of the field, prompting the student to understand under what circumstances the previous theory was judged convincing, and why a new theory is necessary.

Equally important, by recreating the atmosphere of discovery, the historical method inherently brings into the classroom the culture of the field (Conant, 1964; Holton, 1978), informing not only knowledge but also its structure: the deductive logic and reasoning by which knowledge was constructed, older and now obsolete principles and concepts, how they were replaced, the thinking of generations of astronomers - Galili (2010) calls this the periphery of the discipline, as opposed to the discipline's nucleus (axioms and laws) and body (applications).

Finally, research in human cognition shows that learning is facilitated when presented with a contrast between alternatives (Piaget, 1977; Zazkis and Chernoff, 2006; Mansouri et al., 2009; Waxer and Morton, 2012), which is explained within the framework of Variation Theory of Learning (Marton and Booth, 1997; Pang and Marton, 2003; Marton et al., 2004; Marton and Pang, 2008; Orgill, 2012; Bussey et al., 2013; Cheng, 2016). According to variation theory, for learning to occur, some critical aspects of the object of learning must vary while other aspects remain constant. This is exactly how scientists determine cause and effect, mapping the parameter space by exploring one variable at a time while holding all the other variables constant. As a consequence, another reason the historical method is effective as a teaching tool is because, in recreating the narrative that led to the production of knowledge, it recreates the conceptual conflict that necessitated that knowledge (Monk and Osborne, 1997), while also using the tools of the scientific method adapted to the classroom in the form of variational learning.

This paper is structured as follows. In the next section the context of the study is presented, followed by the teaching design (the proposed teaching sequence), including exposition and active learning exercises in the presentation for replicating the method. I conclude with an assessment of student learning and discussion.

\section{Context of the Study}

This method was originally developed as part of a one semester course on dynamical astronomy for physics students in their junior year, at a primarily undergraduate university in California, in 2018. The class had 24 students. I taught it again twice for first-year astronomy PhD students (ASTR 503, "Fundamental Astronomy") at New Mexico State University, in 2019, and 2020. I teach this in two classes of 75 minutes each, as part of a module on Kepler's laws. The first two times were taught in person, the third time online in "flipped classroom" format (King, 1993; the videos are available at https://www.youtube. com/playlist?list=PLatuGW739E01VsAwwqTHKU0tluD9h4c3I). To com-plement the pre-class videos, typeset notes are also provided (available at http://astronomy.nmsu.edu/wlyra/ FundamentalAstronomy/Module3_KeplerLaws_Notes.pdf). The 2019 class had 10 students, which provided a convenience sample (Saumure and Given, 2008) to poll about the 2 nd law.

The fundamental question that guides the module is a question that intrigued humanity for millenia: how to predict the position of the planets? The goal of the module is to understand how Kepler's laws connect to the emergence of modern astronomy, to understand planetary motion, the interplay between theory and observations, and the fundamental importance of observational accuracy. The module on Kepler's laws is done after a module on Spherical Astronomy, so the students are familiar with coordinate systems on the celestial sphere, and how to transform between equatorial and ecliptic coordinates. I also introduce the concept of elongation, the angle between the planet and the Sun. This serves the purpose of introducing Ptolemy's model, which is key to understanding 
Table 1. Initial conditions for the N-body code for computing the position and velocities of "real" Earth and Mars.

\begin{tabular}{lcccc}
\hline Planet & $\begin{array}{c}\text { Semimajor axis } \\
(a)\end{array}$ & $\begin{array}{c}\text { Eccentricity } \\
(e)\end{array}$ & $\begin{array}{c}\text { Inclination } \\
(i)\end{array}$ & $\begin{array}{c}\text { Position } \\
(x y z)\end{array}$ \\
\hline Earth & 1.0000 & 0.0000 & 0 & $(1,0,0)$ \\
Mars & 1.5237 & 0.0934 & 0 & $(1.66601358,0,0)$ \\
\hline
\end{tabular}

the revolutionary character of Kepler's 2nd law. For the junior class I started with Owen Gingerich's celebrated Mars lab (Gingerich, 1983) to find Kepler's lst law in an active learning way (Bonwell and Eison, 1991). For the PhD course, Gingerich's lab was done as a computational exercise. The lab is followed by the geometrical proof that the orbit is an ellipse, again using the historical method, with pre-Newtonian reasoning (Appendix A). In the online version I could not provide drafting tools to each student, so I created a video of the method (available at https://www.youtube.com/watch?v=Ss-nmWFY5Wo\& list=PLatuGW739E01VsAwwqTHKU0tluD9h4c3I\&index=2), and we did active learning in class showing how we would not be able to discriminate between an ellipse and an off-centered circle with the accuracy of common classroom drafting instruments. The lst law lab and instruction set the stage for Kepler's 2nd law.

\section{Teaching Methods}

The historical method was adopted in response to the unsettledness of teaching Kepler's 2nd Law as a post-factum, and with seemingly less importance than the 1st and the 3rd. The lst law, the planets orbit the Sun in elliptical orbits with the Sun at one of the two foci, has a clear and powerful paradigmshifting formulation. Its statement is a direct and unequivocal breaking with the previous cosmological models, of both Ptolemy and Copernicus, that insisted on circular orbits. The 3rd law, the cube of the semimajor axes is proportional to the square of the periods, is formulated as an elementary mathematical statement, and thus conveniently translated into quantitative homework assignments and exams, even at the high school level. In contrast with the 1st and the 3rd, the second law, the radius vector connecting the planet to the Sun sweeps equal areas in equal times, sounds disturbingly turbid to the modern student, its geometric statement a remnant of a pre-calculus era. Transplanting ourselves to Kepler's time by putting aside Newtonian physics and knowledge of conservation of angular momentum, one should ask: why did Kepler care about area? In the junior class in 2018 I asked the students this question. No hands were raised. Not wanting to repeat the usual way of teaching the 2 nd law, and given how other educators also struggle with how to present it (Setyadin et al., 2020), I decided to teach it partially following the historical method to retrace Kepler's original line of thought. In its historical context, Kepler 2 nd law is similar in formulation to the lst law in the sense that it is contrasted to the previous model. The orbit is an ellipse contrasts to the orbit is a circle. Likewise, equal areas at equal times contrasts to equal angles at equal times. The 2 nd law is formulated as a conceptual conflict. For 1500 years, up to Kepler, astronomy insisted not only on circles but also on uniform motion. In Ptolemy's model, to account for the perceived non-uniform motion of a planet, he introduced the equant, which is a point on the line of apsides about which the center of the epicycle does uniform motion. The practicality is that time is given by angle, so the motion of the planet, though non-uniform from Earth's reference frame, is easily parametrized in time. The doctrine of uniform motion was so prevalent that it was an a priori in Copernicus new heliocentric model: to do away with non-uniform motion and cast all planetary movement as uniform circular motion in deferents and epicycles about their centers. Astronomy up to Kepler had it ingrained that there was a reference frame about which a planet sweeps equal angles at equal times. That is the prior model that the 2 nd law contests. Imagine that you never heard about circular orbits before. It would become difficult to understand the paradigm-shifting impact of the lst law. This lack of awareness is precisely the situation a modern student encounters the 2 nd law.

The remainder of this section will be presented for a flipped classroom (King, 1993) format. In this style, content delivery is removed from the in-person classroom time with the students, which is used instead for discussion and active learning. The method here developed to teach Kepler's 2nd law shares similarities with the presentation of the same subject by Holton and Brush (1952). Our method, however, is more mathematically grounded and focuses on the correspondence principle and conceptual conflict between the equant model and $\mathrm{Ke}$ pler's 2 nd law. A drawback of the method is the need to teach the equant model, which many students (as well as instructors) have little familiarity with. However, we minimized the time needed to introduce the model, while also keeping it pedagogical.

\subsection{Presentation of the teaching sequence}

Finding the shape of the orbit is not solving the whole problem of planetary motion. A practical question remains: how to find the planet in the orbit? To better understand Kepler's 2 nd law, let us look at what existed before him. Ancient wisdom insisted in uniform circular motion, because it was their way to understand periodicity. Regularity was found in circles, according to Copernicus (1543), "the only figure that can bring back the past". Although it was apparent that the Sun (or the Earth for that matter) was not the center of the orbit, the shape of the ellipse was out of the reach of their observational accuracy. Non-uniform motion along the orbit was also evident, and a solution was found by Ptolemy, namely, the equant model. Most modern astronomers are not familiar with this model, which is nowadays only of historical importance, so a brief pedagogical exposition is warranted. Let us get to it step by step. Along the way, the students will do active learning exercises to understand Kepler's 2nd law. Like Kepler, we will use Mars in this study, because it is the superior planet of highest eccentricity and, added bonus, also the closest one.

For the exercises presented in this section, the students will use a N-body code of their choice - e.g., Rebound (Rein and Liu, 2012) or Mercury (Chambers, 2012); the plots here shown were calculated with the Pencil Code (Brandenburg et al., 2021) and calculate the orbital evolution of the Earth and Mars. Earth and Mars are test particles (zero mass), so the Sun's position coincides with the barycenter of the system. The results here shown have Earth and Mars initialized at the position and velocities given in Table 1, which are the position and velocity at aphelion. The orbit of the Earth is approximated to zero eccentricity, and the orbits of both planets to zero inclination. In these units an Earth year is $T=2 \pi$. Run the simulation until time $\mathrm{t}=100$, which is approximately 16 Earth years. The students will use this "real" Mars to compare to the different models.

The critical aspect that the instructor should make 
(a) Circle

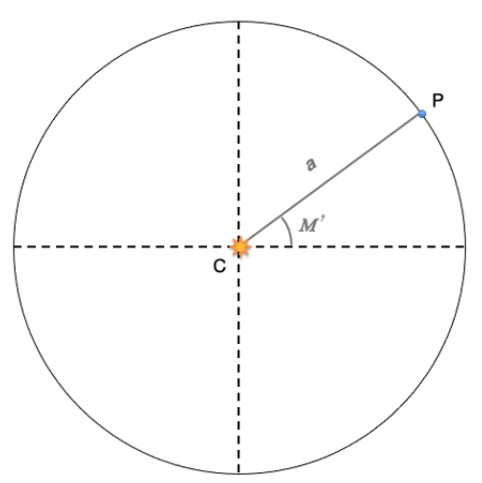

(b) Eccentre

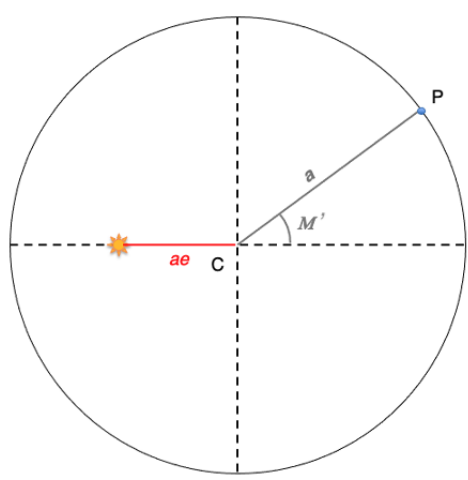

(c) Equant

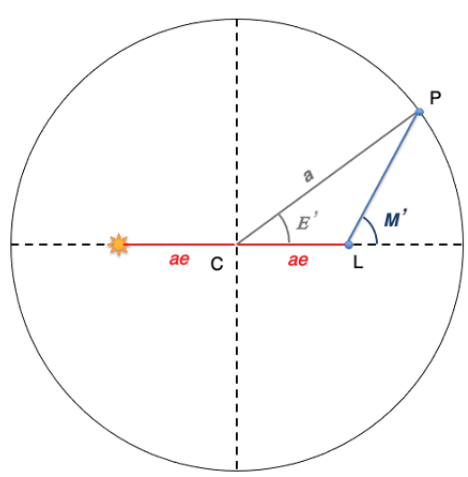

Figure 1. Elements of the different circular orbit models: (a) Sun-centered uniform circular motion about the Sun; (b) Off-centered uniform circular motion about the center; (c) Off-centered uniform motion about the equant (point $L$ ). $M$ is the mean anomaly and $E$ the eccentric anomaly. On (a) and (b) the mean anomaly and the eccentric anomaly are identical.

the students aware of is that the accuracy of ancient observations was $1^{\circ}$, so any model that fits the positions of the planets to one degree accuracy will be deemed acceptable. Otherwise, the model is rejected.

\subsection{Starting simple: a sun-centered, circular model}

Let us assume that planets go in circular orbits, centered at the Sun, in uniform motion (Fig. 1a). At any instant of time, the position of Mars is given by:

$$
\begin{aligned}
& x(t)=a \cos M^{\prime}(t) \\
& y(t)=a \sin M^{\prime}(t)
\end{aligned}
$$

Here $M^{\prime}$ is measured counterclockwise from aphelion; it relates to the mean anomaly $M$ (measured counterclockwise from perihelion) by $M^{\prime}=M-180$. The mean anomaly is $M(t)=n t$, with $t$ meaning time, and $n=\frac{2 \pi}{T}$ is the mean motion, where $T$ is the period of Mars. Here, $M$, the mean anomaly, is equal to both the eccentric and true anomalies. Another critical aspect to raise awareness here is that everything but time in the definition of mean anomaly is constant. Mean anomaly equals time. Mean anomaly is time.

The students will compare this sun-centred circular model with the "real" Mars they calculated from the N-body model. The students should plot the ecliptic longitude of Mars in the sky, which is:

$$
\lambda=\arctan \left(\frac{\Delta y}{\Delta x}\right)
$$

Where $\Delta y=y(t)_{\text {Mars }}-y(t)_{\text {Earth }}$, and $\Delta x=x(t)_{\text {Mars }}-x(t)_{\text {Earth }}$; i.e. the relative position of Mars and the Earth. The students should plot the four subplots of Figure 2. The upper left plot is $\lambda$ vs time for the two models ("real" Mars in red and the circular model of Eqs 1 and 2 in black). The upper right plot is the deviation in $\lambda$ between the model and real Mars. The lower left plot is the bird-eye heliocentric view of the orbits, i.e., $y(t)$ vs $x(t)$. The lower right plot is the bird-eye geocentric view, i.e., $\Delta y$ vs $\Delta x$.

From this exercise, the students should realize that the model does not reproduce either the shape of the orbit or the longitudes. The predicted positions of retrogradations, specifi- cally, are off by as much as 30 degrees from the actual positions of Mars. The students here discern that the circle model cannot be correct, and conclude that the model has to be discarded. The instructor can now introduce variation, fitting other models, and performing the same analysis to assess the adequacy and accuracy of each model in reproducing the observations.

\subsection{The Eccentre}

The circular model failing, the instructor now introduces a new model, the eccentre (Fig. 1b). The critical aspect is centredness: this model merely shifts the position of the center of the orbit away from the Sun by an amount ae, keeping the uniform motion. At any given instant in time, the position of Mars, seen from the Sun, is now given by:

$$
\begin{aligned}
& x(t)=a \cos E^{\prime}(t)+a e \\
& y(t)=a \sin E^{\prime}(t)
\end{aligned}
$$

Where ae is the amount we shift the center away from the Sun. Here $E^{\prime}$, like $M^{\prime}$, is measured counterclockwise from aphelion; it relates to the eccentric anomaly $E$ (measured counterclockwise from perihelion) by $E^{\prime}=E-180$.

The students should compare this model with the "real" Mars. They will plot the same graphs as Figure 2, but now with this model. The results are seen in Figure 3, where the model is shown in cyan. The students realize that the model reproduces the orbit, but it does not reproduce the velocity of Mars. It predicts oppositions and retrogradations still off by 15 degrees. Again, this model cannot be right, and the students discard the model.

\subsection{Non-uniform motion}

Another variation will be introduced, keeping the eccentre, since it reproduces the orbit, but relaxing the idea of uniform motion in order to reproduce the orbital velocity. The instructor now finally presents the equant model (Fig. 1c). To fit the velocity of the planets, Ptolemy added a third device, the equant point, defined as a point on the line of apsides about which the angular velocity of a body on its orbit is constant. This point is point $L$ in Figure 1c. About $L$, the planet, located at $P$, goes around in uniform motion, being described by the angle $M=n t$. The eccentric anomaly $E$ seen from the center of 

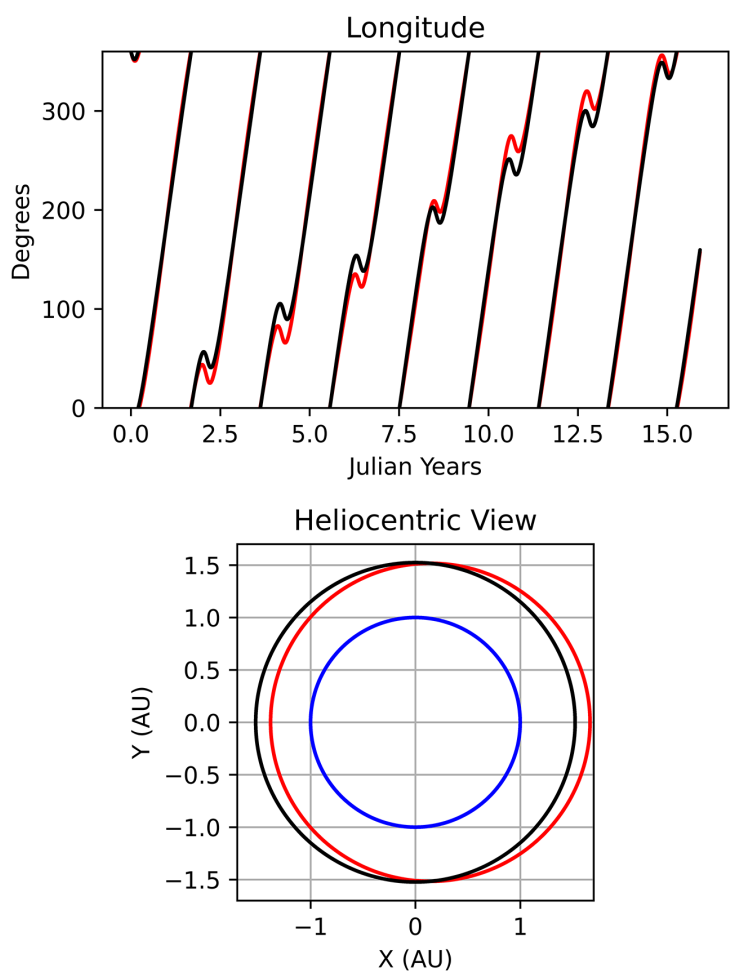

$\Delta$ Longitude
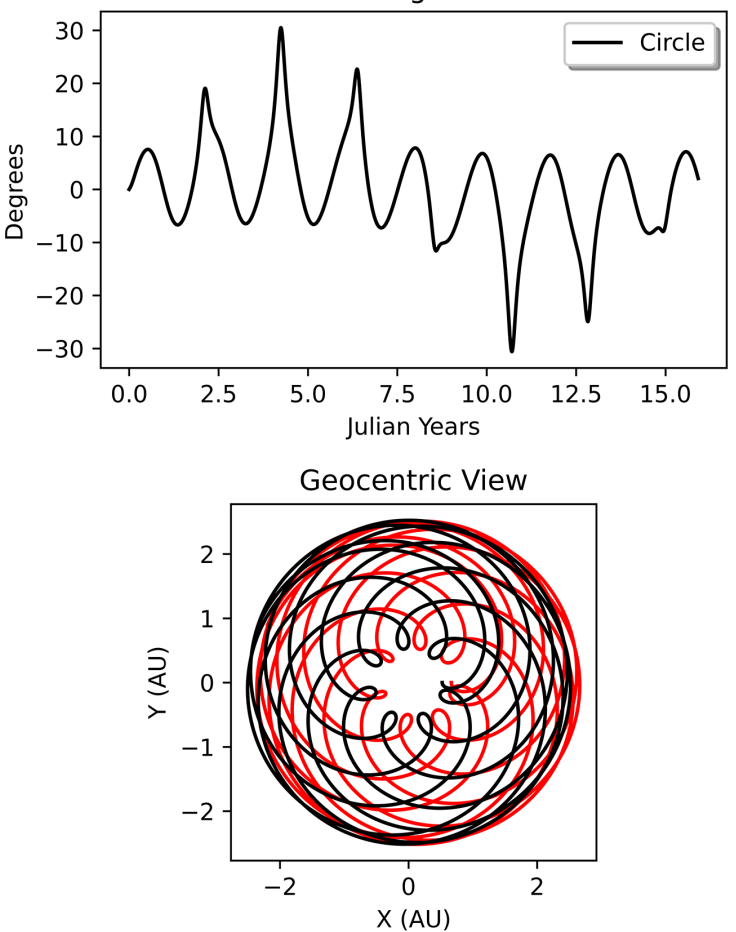

Figure 2. Mars orbit versus a circular Sun-centered orbit model. Upper left plot: Ecliptic longitude vs Time, Mars (red) vs circular orbit (black). The longitudes generally match, except at retrogradations. Upper right plot: Longitude residual. The error amounts to as much as 30 degrees. The circular Sun-centered model is not acceptable. Lower left plot: Heliocentric view of the orbit. Red is mars, blue is Earth, magenta the model. Lower right plot: Geocentric view of the orbit.
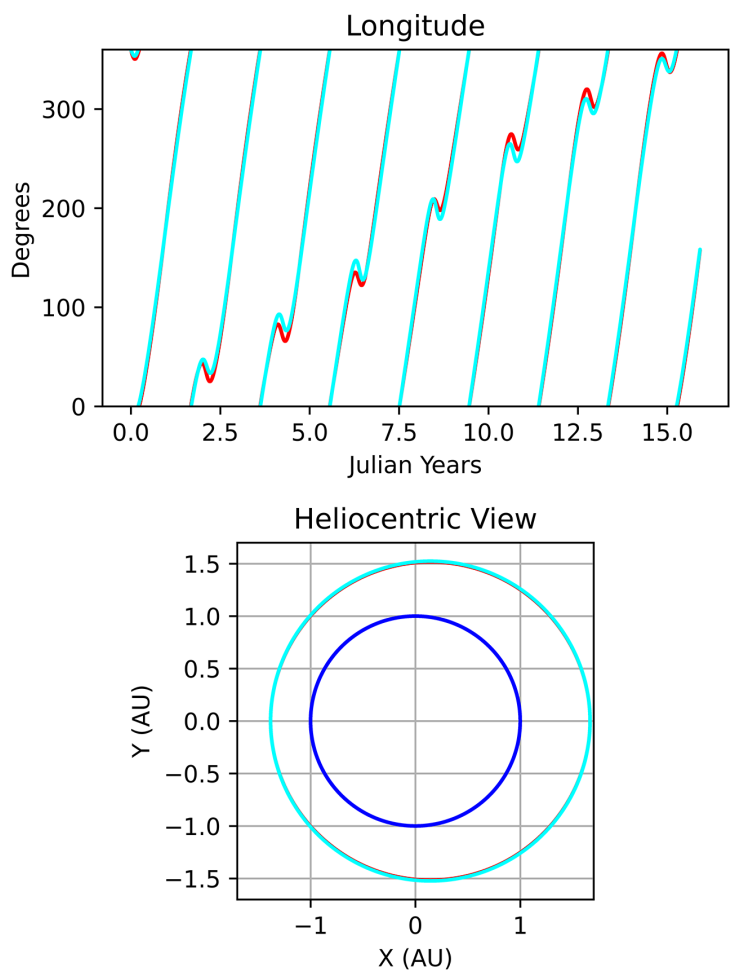

$\Delta$ Longitude
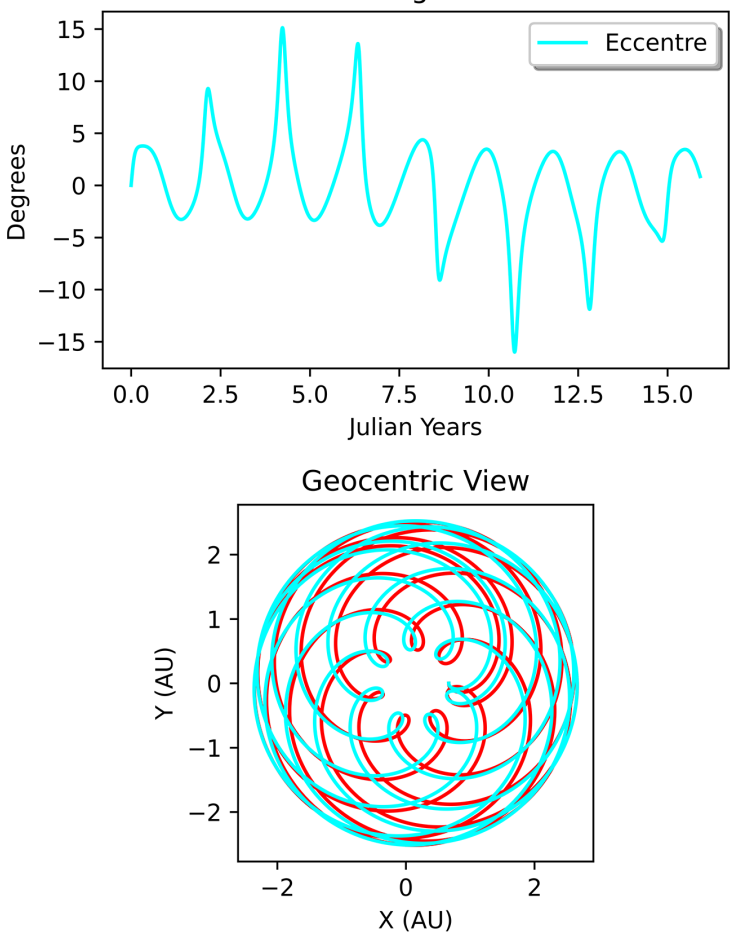

Figure 3. Mars orbit versus off-centered circular orbit model (the eccentre), keeping uniform motion. Upper left plot: Ecliptic longitude vs Time, Mars (red) vs circular off-centered orbit (cyan). The longitudes generally match, except at retrogradations. Upper right plot: Longitude residual. The error is better than the Sun-centered model, but still amounts to as much as 15 degrees. The off-centered model with uniform circular motion is not acceptable. Lower left plot: Heliocentric view of the orbit. Red is mars, blue is Earth, green the model. Lower right plot: Geocentric view of the orbit. 
Table 2. Initial conditions for the $\mathrm{N}$-body code for computing the evolution seen in Fig 5. All planets are massless. The other orbital elements (inclination, longitude of ascending node, and longitude of perihelion) are assumed zero.

\begin{tabular}{lccc}
\hline Planet & $\begin{array}{c}\text { Semimajor axis } \\
(a)\end{array}$ & $\begin{array}{c}\text { Eccentricity } \\
(e)\end{array}$ & $\begin{array}{c}\text { True Anomaly } \\
(f)\end{array}$ \\
\hline Mercury & 0.387098 & 0.20563 & $0^{\circ}$ \\
Venus & 0.723332 & 0.006772 & $180^{\circ}$ \\
Earth & 1.0000 & 0.0167 & $0^{\circ}$ \\
Mars & 1.5237 & 0.0934 & $180^{\circ}$ \\
Jupiter & 5.2044 & 0.0489 & $0^{\circ}$ \\
Saturn & 9.582 & 0.0565 & $180^{\circ}$ \\
\hline
\end{tabular}

the orbit is related to $M$ by noticing that the triangle $\triangle L C P$ has angles $C \hat{L} P=180^{\circ}-M^{\prime}$, and $L \hat{P C}=M^{\prime}-E^{\prime}$. The side $C P$ has length equal to $a$, and the side $L C$ has length equal to ae. The students should find geometrically the relationship between eccentric and mean anomaly in this model. Applying the law of sines:

$$
\frac{\sin \left(M^{\prime}-E^{\prime}\right)}{a e}=\frac{\sin M^{\prime}}{a}
$$

that is,

$$
\sin \left(M^{\prime}-E^{\prime}\right)=e \sin M^{\prime}
$$

Thus, solving for $E$

$$
E^{\prime}=M^{\prime}-\sin ^{-1}\left(e \sin M^{\prime}\right)
$$

At any time, the position of Mars, seen from the Sun, is again given by Eqs (4) and (5), except that now $E^{\prime}(t)$ is non-uniform, given by Eq. (8). Again, the students should graph this model in comparison to "real" Mars. The result is shown in Figure 4. The students visualize that agreement is achieved to within half a degree. At this point the instructor should bring again to the students' focal awareness the critical aspect that Ptolemy did not have accuracy under a degree ( $\mathrm{H} \varnothing \mathrm{g}, 2017)$; thus, the students discern that the equant gives excellent agreement to the observations.

An extra assignment could consist of plotting the results of the equant model to the other planets visible in the pretelescope era, as shown in Figure 5. This figure was computed with an equant for each planet. The "real" planets (Mercury, Venus, Earth, Mars, Jupiter and Saturn) were initialized as shown in Table 2, supposed massless, and alternating perihelion and aphelion.

Each planet has its own equant - which simply reflects the eccentricity of the orbit. Even in the case of Mercury, the planet of highest eccentricity, the agreement with the observations is satisfactory to the degree. The students should also conclude from this exercise how appropriate Mars was as subject of Kepler's analysis. The critical aspect now is that Tycho's observational data was accurate to 2 arcminutes (Høg, 2017). The instructor can ask: "based on this figure you produced, and knowing that Tycho's observations were accurate to two arcminutes, can you tell why Mars was appropriate for elucidating Kepler's laws?" The students should discern that, of the superior planets, Mars is the one whose deviation more blatantly disagreed with the prevailing model. Venus, Jupiter, and Saturn deviate by less than 2 arcminutes, within the accuracy of Tycho's data. Mercury, never too far from the Sun, is simply too difficult to observe.

\subsection{Optional}

The instructor may want at this point to do a parenthetical comment, returning for a moment to modern scientific parlance, and noting that the equant model is a model accurate to first order in eccentricity (Hoyle, 1973; Murray and Dermott, 1999). At higher eccentricities the equant model will again start to deviate significantly from the observations. Figure 6 shows a hypothetical planet of eccentricity $e=0.45$. The equant model is off by more than 10 degrees. While for $e \sim 0.2$, like Mercury's orbit, the model is satisfactory down to 40 arcmin accuracy, for $e=0.45$ one would need higher order corrections. Kepler's 2 nd law is the full solution.

\subsection{The ellipse has no equant}

Ptolemy's solution had a very practical function. Given a point in the line of apsides upon which the planet sweeps equal angles in equal times, the orbit can be parametrized, as given by Eqs. (4) and (5) with E given by Eq. (8). Kepler had two problems. First, Tycho's observations of Mars, accurate to 2 arcmins, did not allow for the 30 arcmin error given by the equant model. Second, his ellipses, with the planet speeding nearing perihelion and slowing down nearing aphelion begged the question: what is the equivalent to the equant? What is the point about which a planet sweeps equal angles in equal times? Where is the point along the line of apsides that we can say that angle equals time? As it turns out, Kepler's quest to answer this question culminated with his 2 nd law, that demolished the idea of uniform motion. The answer is: there is no equant. For the ellipse, there is no point about which an observer will see equal angles at equal times. Kepler's first law does away with the epicycle. Kepler's second law does away with the equant. This subsection is the one directly from Kepler's Astronomy Nova. I taught it in detail twice, before realizing that the level of detail is unnecessary. What the students should know is that Kepler tried to find the equant for the ellipse and failed to find it In trying to find out the location of Mars' equant, Kepler again made use of Tycho's data. He took four observations of Mars in opposition, at times $t_{1}, t_{2}, t_{3}$, and $t_{4}$, corresponding to mean anomalies $M_{1}, M_{2} M_{3}$, and $M_{4}$. The equant would be the unique point on the line of apsides whence Mars is seen at these angles (Fig. 7).

Yet, Kepler's best fit with an "ellipse equant" model was incompatible with the observations by 8 arcminutes, which was inadmissible by Tycho's 2 arcminute accuracy. Kepler had to go back and question his assumptions. But the assumptions were minimal. They amounted to:

1. Mars orbits the Sun;

2. Tycho's observations are reliable;

3. The equant exists.

(1) and (2) were beyond doubt correct. The conclusion was astonishing. The equant, a staple of astronomy for 1500 years, cannot exist. Kepler started this analysis by asking the question: where is the equant? And the answer was: there is no equant. There is no point about which we can say the planet sweeps equal angles at equal times. Uniform motion does not exist. Time is not given by angle. This is the aspect of the second law that should be emphasized: it rules out 1500 years of the paradigm of uniform motion. 

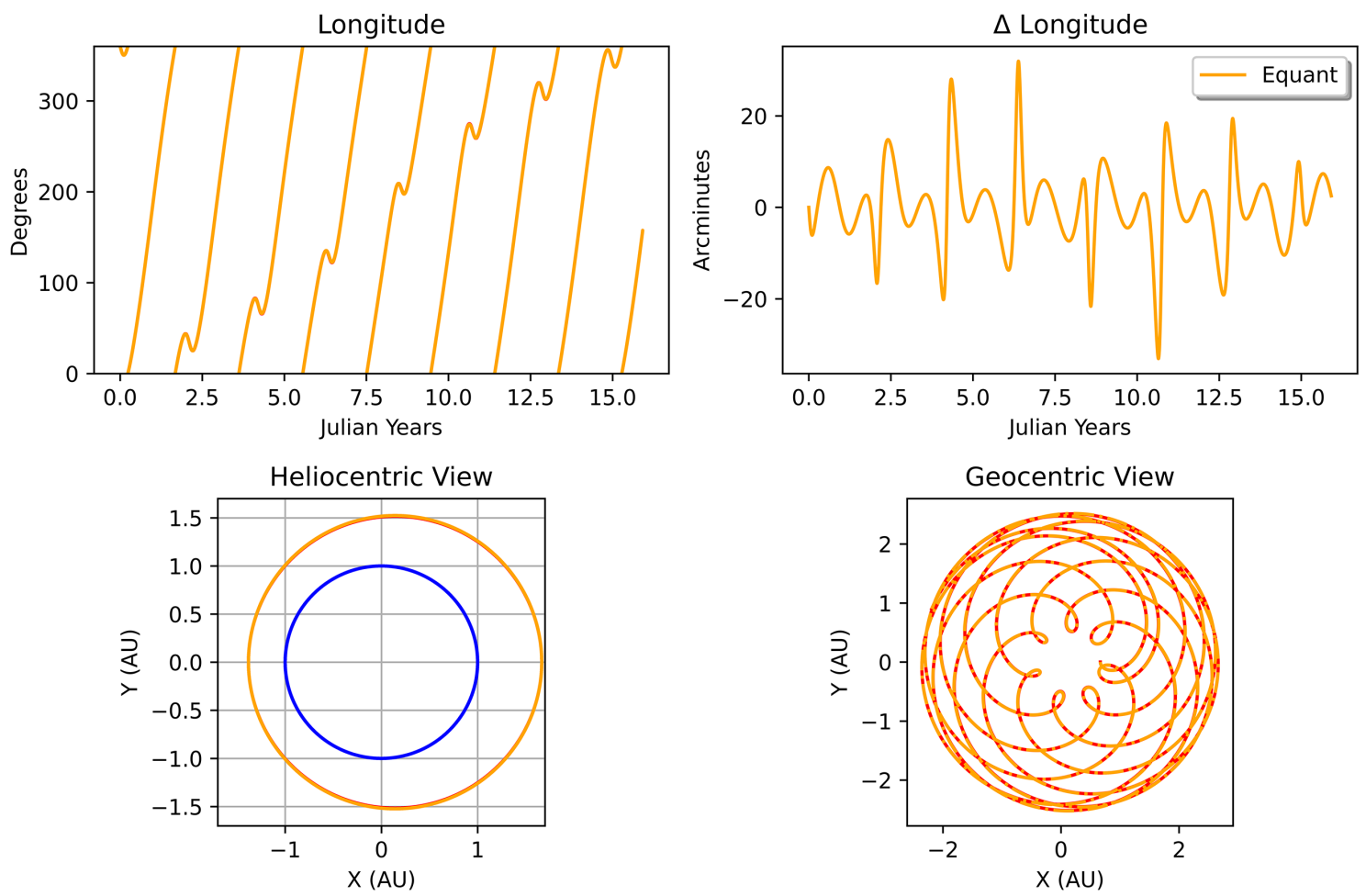

Figure 4. Mars orbit versus off-centered circular orbit model, with uniform motion about the equant. Upper left plot: Ecliptic longitude vs time, Mars (red) vs equant model (orange). Upper right plot: Longitude residual. The error is at most 30 arc minutes. Ptolemy's accuracy was 1 degree. The model is acceptable. Lower left plot: Heliocentric view of the orbit. Red is mars, blue is Earth, orange the equant model. Lower right plot: Geocentric view of the orbit. The equant model reproduces location and time of retrogradations.

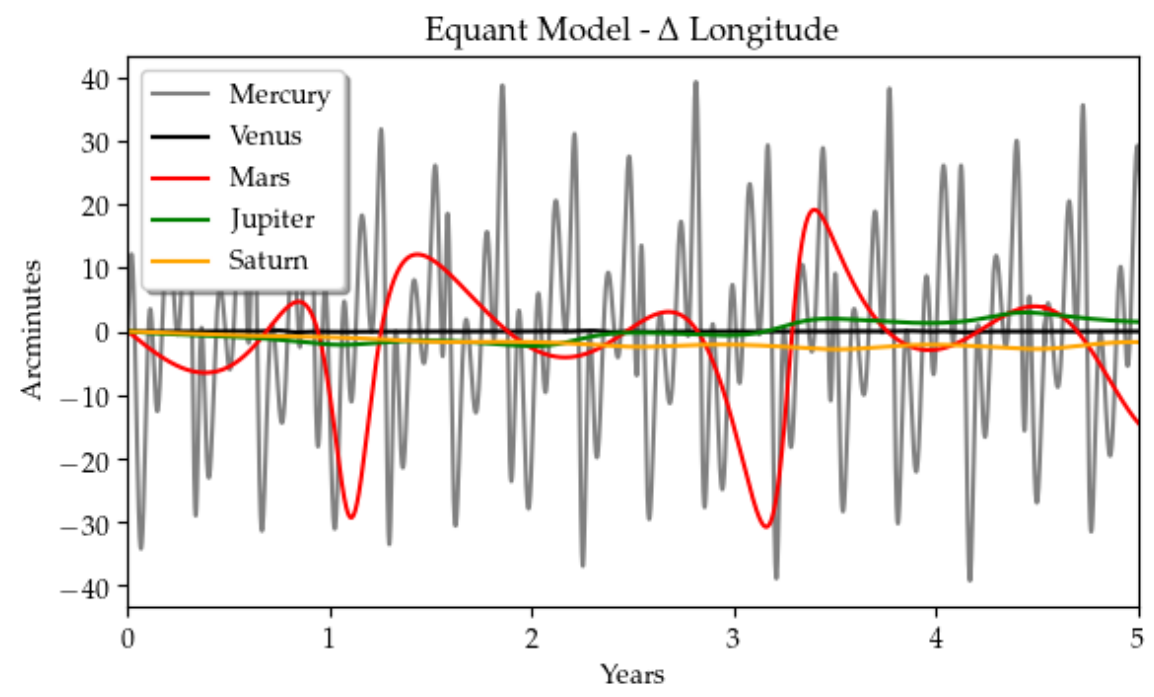

Figure 5. Residuals of the equant model for each planet. The residuals reflect orbital eccentricity. Even for Mercury, the most eccentric planet $(e=0.2)$, the equant agrees with the observations down to 40 arcminutes. 

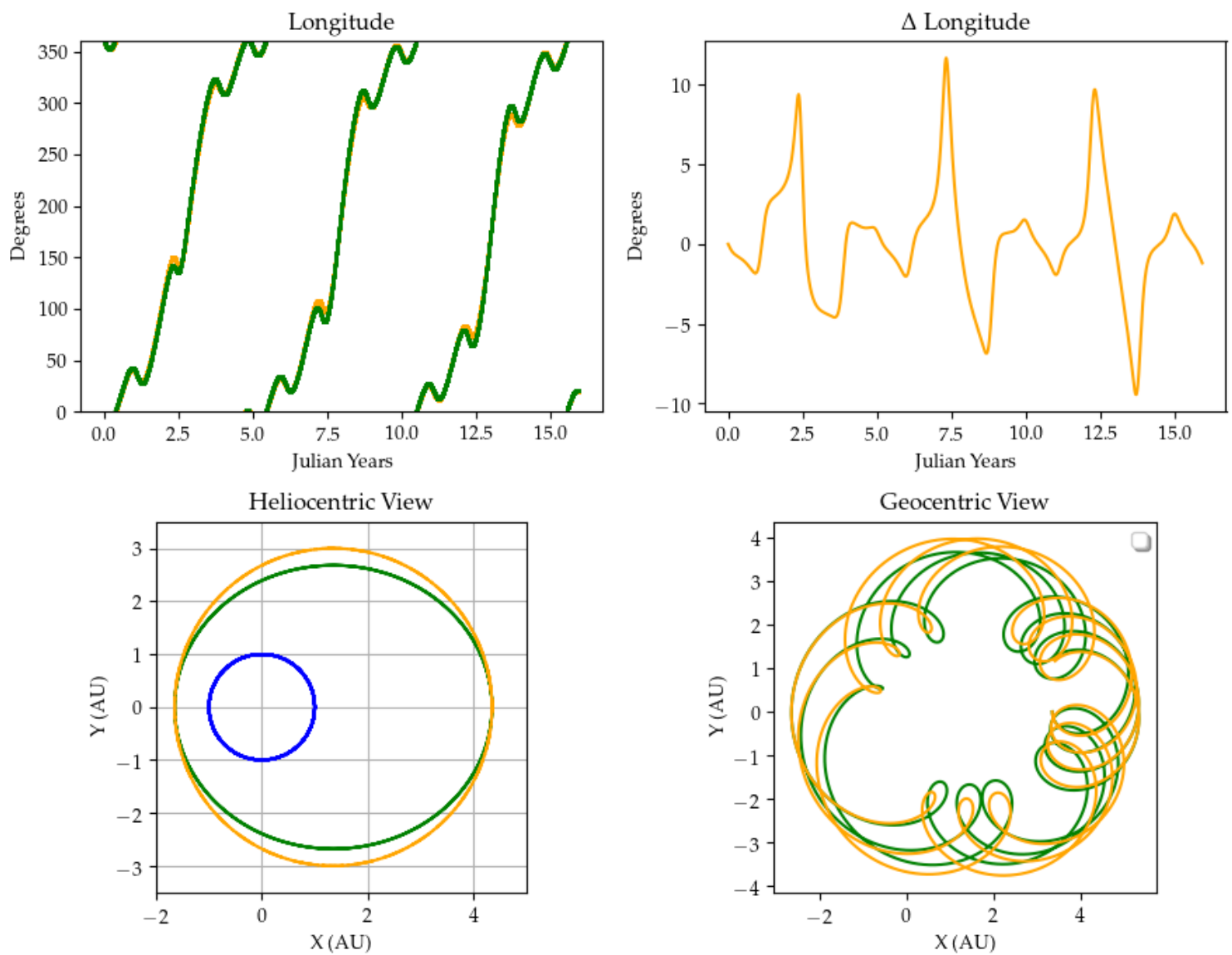

Figure 6. Validity of the equant model. A hypothetical planet (green) with orbital eccentricity 0.45 , The equant model (orange) does not reproduce it well anymore. The equant is a model accurate only to lst order in eccentricity.

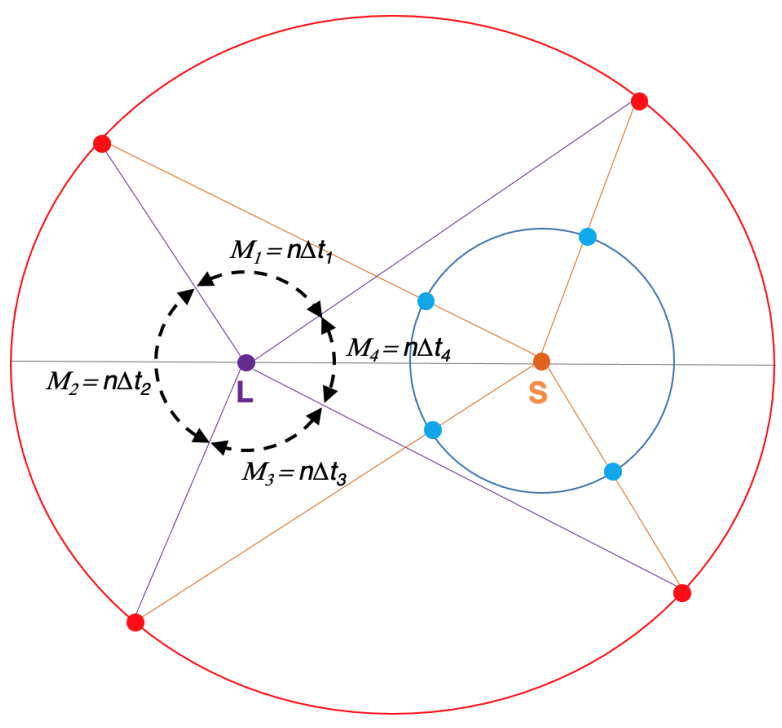

Figure 7. Kepler's method to determine the location of Mars' equant in an elliptic orbit, using four observations of Mars in opposition. The four dots in each orbit represent the four observations; the motion is counterclockwise. Because the Sun (S), Earth (blue orbit and dots), and Mars (red orbit and dots) are aligned, the orange lines intersect at the Sun. Timing the observations yields the mean anomalies $M_{1}, M_{2}, M_{3}, M_{4}$. Kepler then looked for the point $L$ in the line of apsides where Mars would be seen at exactly these angles, failing to find it. The model could not be reconciled with the observations by 8 arcminutes, inadmissible by Tycho's 2 arcminute accuracy, forcing him to discard the equant model. The eccentricity of Mars' orbit is highly exagerated $(\sim 0.4$ instead of $\sim 0.1)$ for clarity.

\subsection{Time is equal area}

Kepler had disproved fifteen centuries of "equal angles at equal times". That still leaves the problem of how to find the eccentric anomaly as a function of time. In looking for something that could be a measurement of time, Kepler stumbled on what this something was.

Because planets are slower when far from the Sun and faster when close, Kepler reasoned that the velocity was inversely proportional to the distance, $u \alpha \frac{1}{r}$. If that is the case, one can multiply both sides by time and write the proportionality:

$$
u r t \alpha t
$$

The quantity in the left-hand side, whatever it is, is linearly proportional to time. It is the "something" sought in equal "something" at equal times. But what is its interpretation?

The product $u t$ is the length of the arc swung by the planet. If the time is infinitesimal, $t \rightarrow d t$, the arc is infinitesimal, $d l=u d t$. Then the Sun, the planet's position at $t$ and its position at $t+d t$ form a triangle, of area $r d l / 2$. Comparing to Eq. (9), the quantity $u r d t=r d l$ that is linearly proportional to time is thus the area As a planet orbits the Sun, the area it sweeps is proportional to time. Mean anomaly is not given by an angle. Mean anomaly is given by an area. 


\subsection{Kepler's Equation}

At this point the class becomes finding the mathematical statement of Kepler's equation. The critical aspect to focus on is that the 2 nd law is quantitative, relating the eccentric anomaly of the planet (and consequently its true anomaly) to the mean anomaly. The students have already studied in the lst law the geometry of the ellipse (Appendix A).

The mean anomaly is proportional to the area. The question then is, how to compute the area? Kepler did not know calculus, so he could not calculate the area by summing the infinite infinitesimal distances. But Kepler was an excellent geometer. After he discovered that the orbit was an ellipse, he used the geometry of the ellipse to find out the area. Most students do not know a proof that the area of the ellipse of semimajor axis $a$ and semiminor axis $b$ is $\pi a b$, so a short proof of it using integration is shown in Appendix B.

Given that the planet sweeps equal areas at equal times, a relation between mean anomaly (time) and area can be established. A planet sweeping equal areas at equal times will, within a time $t$, sweep an area

$$
A_{\text {sector }}=\pi a b \frac{t}{T}
$$

where $T$ is the orbital period. The question is, what is the area of the sector? Consider Figure 8 . The area swept from perihelion $(X)$ to point $P$ is the area of the elliptic sector $A_{S P X}$. We can relate it to the area of the circular sector $A_{S Q X}$ by using the relation $\mathrm{PH} / \mathrm{QH}=b / a$

$$
A_{S P X}=\frac{b}{a} A_{S Q X}
$$

The elliptic sector SQX can be broken down as the circular sector CQX, minus the triangle $\triangle C S Q$

$$
A_{\text {SQX }}=A_{C Q X}-A_{C S Q}
$$

The circular sector CQX comprises an angle $E$ of the full $2 \pi$ circle, so its area is

$$
A_{C Q X}=\pi a^{2} \frac{E}{2 \pi}
$$

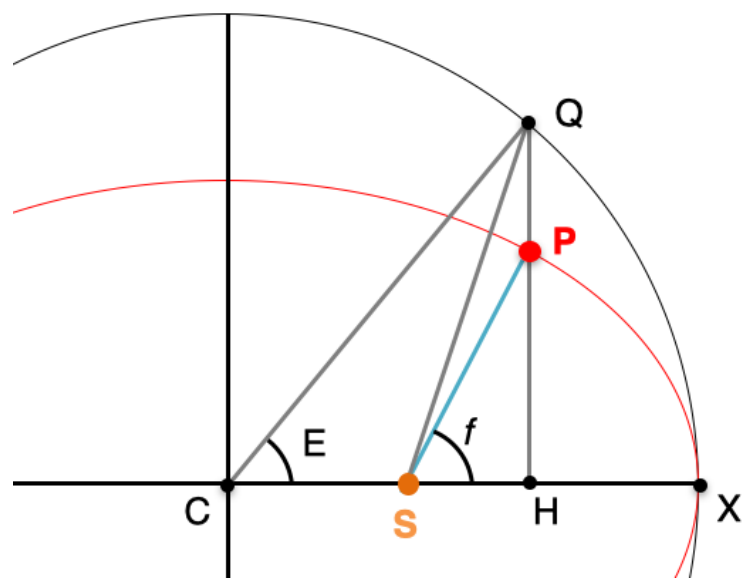

Figure 8. The true anomaly $f$ is the angle, with vertex at the Sun, from perihelion to the planet. The eccentric anomaly $E$ is the angle with vertex at the center of the orbit, from perihelion to the planet. Given the geometry of the ellipse, $\mathrm{PH} / \mathrm{OH}=b / a$, where $b$ is the semiminor axis and $a$ is the semimajor axis.
As for the triangle $\triangle C S Q$, its base is $C S=a e$, and height $Q H=$ $a \sin E$

$$
A_{C S Q}=\frac{1}{2}(a e)(a \sin E)
$$

We thus find the area of the elliptic sector SPX,

$$
A_{S P X}=\frac{a b}{2}(E-e \sin E)
$$

But because area equal time, $A_{S P X}=\frac{\pi a b t}{T}$. Equating both,

$$
2 \pi \frac{t}{T}=E-e \sin E
$$
Thus,

Given $n=\frac{2 \pi}{T}$, the left hand side is $M=n t$, the mean anomaly.

$$
M=E-e \sin E
$$

This result is known as Kepler's equation. It is a direct consequence of Kepler's 2 nd law, and can also be seen as Kepler's 2 nd law itself. The left-hand side is time. The right-hand side is area. After teaching Kepler's 2 nd this way, I gave as homework assignment a computational exercise where students had to predict the timing and location of Mars' next opposition, given the orbital elements and the current position of Mars and the Sun. I tested the method in both traditional and flipped classroom (King, 1993) environments. In the latter, the lab was started in an online class (taught during the 2020 pandemic of COVID19), with the students sharing a python jupyter notebook via simultaneous video conferencing while freely debating. The method is thus in line with active learning (Bonwell and Eison, 1991), constructivist learning theory (Simon, 1995), and structured in a multimodal framework, featuring: videos, which are optimal for the visual/audio learners; notes, aimed at the reader learners; team-based learning, which helps the social learners; and coding exercises for the logical/math learners.

\section{Assessment of Student Learning}

A pre-and post- test was used in the graduate class administrated in 2019; the class had 10 students. The pre-test questionnaire had questions pertinent to all material covered in Fundamental Astronomy, among which one of the questions was "What is the relevance of area in Kepler's 2 nd law?", to be answered discursively. The post-test was administrated after both Kepler's Laws and Celestial Mechanics modules, 3 weeks after Kepler's 2nd law was taught.

All students had the qualitative understanding of the law, as expected from graduate students in a major astronomy research institution, yet no one tied it to the crucial fact of finding the true anomaly. Post-instruction, the answers varied little from the pre-test, with $70 \%$ of the students repeating the "equal areas equal times" response of the pre-test. 30\% showed a different answer: that area "allows position of the planet to be determined", "is time", and "shows there is no reference point from which an object appears to orbit equal angles in equal times". Perhaps the question of the pre-and-post test could have been better phrased. In contrast, anecdotal feedback I received so far, as well as the degree of student comprehension on dealing with Kepler's equation, supports the approach. One student in particular approached me to say they found the lab "inspiring" and "really interesting", adding in particular that they liked the way 
the class was taught, going through the historical development of the ideas and the critical thinking involved. One student, after the Mars opposition computational exercise, stated that "now I finally understand Kepler's 2 nd law and how to use it". Another student said they liked how the class was taught "like research". Yet another student declared "mad respect" for Kepler after the module.

\section{Conclusion}

In this work I constructed a way to teach Kepler's 2 nd law based on the historical method. The perceived benefits of the approach are enumerated below.

1. It frames the teaching in terms of a conceptual conflict, which research in human cognition shows is conducive to more effective learning (Piaget, 1977; Waxer and Morton, 2012). The conflict is between the equant model (equal angles) and Kepler's 2nd law (equal areas).

2. Variational learning (Marton and Booth, 1997) is naturally brought to the classroom. In a scientific experiment, the impact of a variable is isolated by controlling its change while holding all the other variables constant, and analyzing the effect of the change. According to variational theory, this parallels how students construct learning. In the case in question, students explore different models until a match between data and model is achieved to observational accuracy. The different models hold one aspect constant while varying others (Sun centered/off-centered, and uniform/non-uniform motion).

3. The method uses the correspondence principle (Bohr, 1920), making the students understand the validity and limits of the equant model in its own historical framework, as a valid model that reproduces the observations up to about half a degree. The students understand why it worked (a model accurate to first order in eccentricity) and why it had to be discarded (when observational accuracy became better than the 40 arcminute accuracy given by the model). Data of worse quality would not have been able to discern between the equant model and Kepler's 2nd law. As such, the approach also emphasizes to the students the paramount importance of observational accuracy.

4. The proposed approach highlights the revolutionary character of Kepler's 2nd law: instead of repeating "equal areas in equal times" instructors can instead say "contrary to 1500 years of astronomical lore, there is no such thing as equal angles in equal times. Kepler's lst law discards the epicycle. Kepler's 2 nd law discards the equant. Area is how you measure time and hence how you find the planet."

5. The approach is also rooted in mathematical grounding, as "time = area" is stated not only through geometrical illustrations, but by Kepler's equation (Eq. 17). Students can then manipulate it quantitatively, as usually done for the 3rd law.

6. By recreating the atmosphere of discovery, the method also frames the class in terms of cultural teaching (Matthews, 1989; Galili, 2010), bringing into the classroom the culture of astronomy. It is a narrative method that reveals the inner workings of the minds of the pioneers of the discipline, allowing their own voice to be brought into the classroom. For instance, Kepler famously wrote, in trying to locate the equant (Fig 7): "If this wearisome method has filled you with loathing, it should more properly fill you with compassion for me as I have gone through it at least seventy times at the expense of a great deal of time." That is a feeling that many a graduate stu- dent can empathize with. Combining human reason and emotion with the timeless elements of paradigm-shifting research, namely, tension between theory and new data, new data leading to a new theory, the new theory corresponding to the previous theory in its limit of applicability, this is a method that humanizes science and creates a bridge between a student experience and that of the greatest names in the history of the field.

Finally, on the limitations of the method, it has been said that the inquiry method, of which the historical method is a subset, is too difficult for any but the brightest students and that by teaching discarded ideas it is prone to causing confusion (Welch et al., 1981). Indeed, the method has been tested in upper division undergraduate and graduate studies only, so its effectiveness at lower division or general education courses is unconstrained. Also, because Kepler's equation is transcendental, this method is best used in graduate curricula, where computational techniques are more routinely applied. Another criticism is that scientists are not historians and, by attempting to teach history of science we incur into the danger of teaching bad history (Matthews, 1989). Also, as stated by (Ausubel, 1968) "the most important factor influencing learning is what the learner already knows". Indeed, resilience of previous conception is observed, as $70 \%$ of students repeated the pre- and post-test answer. The post-test was given after 3 weeks of instruction and after teaching Celestial Mechanics. It is unclear if the time elapsed, the introduction of Newtonian physics, or the phrasing of the question influenced this result.

\section{Conclusion}

I would like to acknowledge productive conversations with Joshua Tan, Mordecai Mac Low, and Roberto Pimentel. A first draft of this paper greatly benefited from comments by Anna Danielsson.

\section{Author Biography}

Wladimir Lyra is an Assistant Professor of Astronomy at New Mexico State University whose scholarship focuses on planet formation.

\section{Appendix A: Elliptical orbits (Kepler's 1st law)}

In this appendix, the instructor guides the demonstration that the shape of the orbit is an ellipse. It works as active learning, with the instructor giving to each student a sheet with the elliptic shape, and a set of drafting tools. This appendix is written in teacher's voice, guiding how to draw Figure 9 step by step. The order: is $A P$, circle centered at $A, P H$, circle centered at $B$, $P Q, B Q, \beta=H \hat{B} Q, Q K, A K, A K Q R$, and finally $\beta=A \hat{B} R$. A video is available at https://www. youtube.com/watch?v=Ss-nmWFY5Wo\& list=PLatuGW739E0IVsAwwqTHKU0tluD9h4c3I\&index=3.

Having found the orbit, Kepler had no idea what geometrical shape it corresponded to. Yet, Kepler realized, through geometry, some properties this shape had. Consider Figure 9 (at this point with the only the red curve drawn, points $B, C, P$, major and minor axes; the focus $A$ can be pre-drawn, or it can be found with the compass, from point $F$ and striking the line of apsides with length $B C$, the semimajor axis). The Sun is at point $A$, and Mars at point $P$. The segment AP, of length $r$, is the radius vector from the Sun to the planet. The orbit is the red curve, which, a priori, we do not know what shape it corresponds to. 


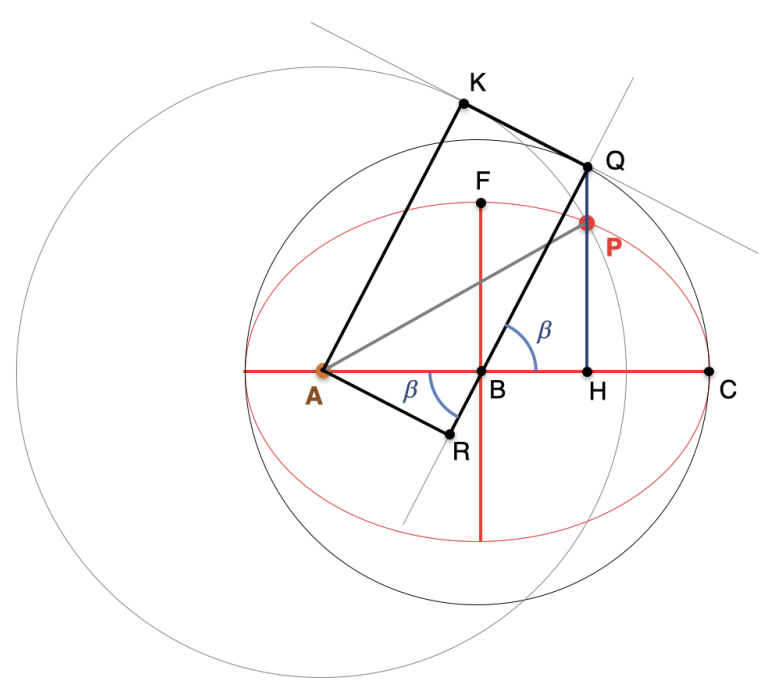

Figure 9. Kepler's method to find the curve corresponding to Mars' orbit. The crucial insight was to realize that a perpendicular to $B Q$ at $Q$ is tangent to the circle centered at the Sun $(A)$ and with radius $r$ equal to the radius vector that joins the Sun and the planet $(\mathrm{P})$. The eccentricity of Mars' orbit is highly exagerated for clarity.

\section{Some elements are:}

1. The aphelion is point $C$;

2. The line of apsides is bisected at point $B$, the geometric center of the curve;

3. The length of the segment $B C$ is by definition $a$;

4. The length of the segment $A B$ is by definition ae; $e$ is the eccentricity of the ellipse, but we do not know that yet. So far $e$ is an adhoc constant, the factor by which we need to move the Sun away from the center.

What we want to find is $A P$, the radius vector of the orbit (draw AP). Algebraically, one would call it $r$ and try to find a mathematical relationship for it. Geometrically, one draws a circle. This circle is centered at $A$ and has radius $A P$ (draw circle). We do not need to find exactly $A P$; if we find the radius of this circle, at any angle, we find the length of the radius vector. We trace the circle in the hope that a geometric coincidence that helps tell what the length $r$ is becomes obvious.

Another way to find the shape of the curve is to find the coordinates $x$ and $y$ of the planet, and uncover their mathematical relation. We find the coordinate $x$ by drawing the perpendicular from $P$ to the line of apsides, defining point $H$ (draw $P H$ and point $H$ ). The coordinates of $P$ are $x=B H$ and $y=P H$.

Next, we define the eccentric anomaly. For that we draw the circumscribed circle, of center $B$ and radius $B C=a$ (draw circle). We prolong the line $P H$ until it intersects the circumscribed circle at point $Q$ (draw $P Q$ ). The eccentric anomaly is $E=A \hat{B} Q($ draw $B Q$ ). We will also define the auxiliary angle $\beta=E-180^{\circ}=H \widehat{B Q}$, (draw $\beta$ ). Given the triangle $\triangle B H Q$, the coordinate $x$ is $B Q \cos \beta$. Given $B Q=a$, we found the first coordinate.

$$
x=a \cos \beta
$$

As for $y$, the triangle $\triangle A H P$ can be used. It is a right triangle where $A P=r$ is the hypotenuse; the catheti are $P H=y$, and $A H=A B+B H=a e+x$. Thus,

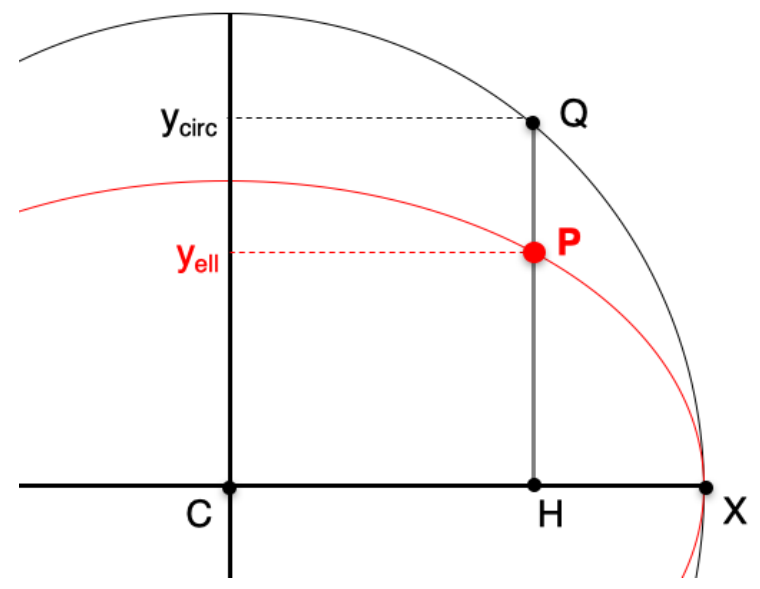

Figure 10. The ratio $\mathrm{PH} / \mathrm{QH}=y_{\text {ell }} / y_{\text {circ }}$ is equal to $b / a$, where $b$ is the semiminor axis and a the semimajor axis of the ellipse.

$$
y^{2}=r^{2}-(a e+x)^{2}
$$

Eq 18 gives the value of $x$, but the value of the radius vector $r$ is so far unknown. Kepler found $r$ in an ingenious way. He realized something curious: the perpendicular to $B Q$ at $Q$ is tangent to the circle of center $A$ and radius $r$. (prolong $B Q$ and draw the perpendicular)

Let $K$ be the tangential point (draw $K$ ). Since $A K$ is a radius (draw $A K$ ), then $A P=A K=r$. So, if we find $A K$, we find the value of $r$. Because $Q K$ is tangent to the circle, $A \hat{K} Q$ is a right angle. Kepler then prolonged the radius $B Q$ to construct the rectangle $A K Q R$ (draw the rectangle and define $R$ ). Because this is a rectangle, $A K=Q R=r$. The length $Q R$ is the sum of the radius $(B Q=a)$ and the length $B R$. This length is given by the right triangle $\triangle A R B$. The hypothenuse is $A B=a e$, and the cathetus $B R=$ ae $\cos \beta$ (draw $A \hat{B} R=\beta$ ). We find thus $A P=B Q+B R$, or $r=a+a e \cos \beta$. Given $\beta=E-180^{\circ}$.

$$
r=a(1-e \cos E)
$$

Having found the radius vector, we substitute Eq. 20 and Eq. 18 into Eq. 19, finding

$$
y^{2}=a^{2}\left(1-e^{2}\right) \sin ^{2} E
$$

We can then write $\cos ^{2} E=x^{2} / a^{2}$, and $\sin ^{2} E=y^{2} /\left[a^{2}\left(1-e^{2}\right)\right]$ and invoke the trigonometric equality $\sin ^{2} E+\cos ^{2} E=1$ to find the relationship between the coordinates

$$
\frac{x^{2}}{a^{2}}+\frac{y^{2}}{a^{2}\left(1-e^{2}\right)}=1
$$

This is the equation of an ellipse. The semimajor axis is a, and the semiminor axis is $b=a\left(1-e^{2}\right)^{1 / 2}$.

\section{Appendix B : Short proof of the area of the ellipse}

Consider Figure 10. The main insight is that $P H / Q H=b / a$, which is seen because $C Q=a$, and thus $Q H=a \sin E$, and we have already proven $(E q 21)$ that $P H=a\left(1-e^{2}\right)^{1 / 2} \sin E=b \sin E$. 
The area $A_{C}$ of the circle is 4 times the area of the quadrant. The area of the quadrant can be found by integrating the vertical distances $y_{c}$ from $x=0$ to $x=a$.

$$
A_{c}=4 \int_{0}^{a} y_{c} d x
$$

Given $y_{c}=a \sin E$ and $x=a \cos E$, then $A_{C}=\pi a^{2}$, as expected. The area of the ellipse is

$$
A_{e}=4 \int_{0}^{a} y_{e} d x
$$

Because we can write $y_{e}=b / a y_{c}$, then

$$
A_{e}=4 \frac{b}{a} \int_{0}^{a} y_{c} d x=\frac{b}{a} A_{c}=\pi a b
$$

\section{References}

Aiton, E. J. (1969). Kepler's second law of planetary motion. Isis, $60(1): 75-90$

Aktan, D. Ç. and Dinçer, E. O. (2014). examination of pre-service science teachers' understanding levels of kepler's laws with ranking task questions. Journal of Baltic Science Education, 13(2):276. http://oaji.net/articles/2015/987-1437063463. pdf.

Ausubel, D. (1968). Educational psychology: a cognitive view. New York: Holt, Rinehart \& Winston.

Boccaletti, D. (2001). From the epicycles of the greeks to kepler's ellipse - the breakdown of the circle paradigm. In Cosmology Through Time Ancient and Modern Cosmology in the Mediterranean Area, Monte Porzio Catone (Rome), Italy, June 18-20, 2001.

Bohr, N. (1920). Über die serienspektra der elemente (on the spectral series of the elements). Zeitschrift für Physik, 2(5):423-469. https://doi.org/10.1007/BF01329978.

Bonwell, C. C. and Eison, J. A. (1991). Active Learning: Creating Excitement in the Classroom. 1991 ASHE-ERIC Higher Education Reports. ERIC. https://files.eric.ed.gov/fulltext/ ED336049.pdf.

Brandenburg, A., Johansen, A., Bourdin, P. A., Dobler, W., Lyra, W., Rheinhardt, M., Bingert, S., Haugen, N. E. L., Mee, A., Gent, F., et al. (2021). The pencil code, a modular mpi code for partial differential equations and particles: multipurpose and multiuser-maintained. Journal of Open Source Software, 6(58):2807.

Bussey, T. J., Orgill, M., and Crippen, K. J. (2013). Variation theory: A theory of learning and a useful theoretical framework for chemical education research. Chemistry Education Research and Practice, 14(1):9-22.

Carroll, B. W. and Ostlie, D. A. (2007). An introduction to modern astrophysics and cosmology. Pearson, San Francisco.

Chambers, J. E. (2012). Mercury: A software package for orbital dynamics. Astrophysics Source Code Library, pages ascl1201.

Cheng, E. W. (2016). Learning through the variation theory: A case study. International Journal of Teaching and Learning in Higher Education, 28(2):283-292.

Coelho, R. L. (2009). On the concept of energy: how understanding its history can improve physics teaching. Science \& Education, 18(8):961-983. https : //doi.org/10.1007/ s11191-007-9128-0.

Conant, J. B. (1964). Case studies in experimental science. Harvard university press.
Copernicus, N. (1543). On the Revolution of the Heavenly Spheres.

de Cayeux, A. and Brunier, S. (1983). Les planètes. Ciel et Terre, 99:29.

Dixon, F. A., Prater, K. A., Vine, H. M., Wark, M. J., Williams, T., Hanchon, T., and Shobe, C. (2004). Teaching to their thinking: A strategy to meet the critical-thinking needs of gifted students. Journal for the Education of the Gifted, 28(1):56-76 https://doi.org/10.1177/016235320402800104.

Duhem, P. (1954/1906). The aim and structure of physical theory. Princeton University Press, Princeton: NJ.

Galili, I. (2010). History of physics as a tool for teaching. In Vicentini, M. and Sassi, E., editors, Connecting research in Physics Education with Teachers Education., pages 153-166. I.C.P.E. book.

Gingerich, O. (1983). Laboratory exercises in astronomy-the orbit of mars. Sky and Telescope, 66:300.

Guimaraes, L. A. and Fonte Boa, M. (2006). Fisica Volume 1: Mechanica. 3rd Edition. Hipermidia Guys, Brazil.

Halliday, D. and Resnick, R. (2020). Physics for Students of Science and Engineering. John Wiley \& Sons Inc.

$\mathrm{H} \varnothing \mathrm{g}, \mathrm{E}$. (2017). Astrometric accuracy during the past 2000 years. arXiv preprint arXiv:1 707.01020. https://arxiv.org/ abs/1707.01020.

Holton, G. (1978). On the educational philosophy of the project physics course. In The Scientific Imagination: Case Studies. Cambridge University Press, Cambridge.

Holton, G. and Brush, S. (1952). Introduction to concepts and theories in physical science. 3rd edition: Holton, G., Brush, S. \& Evans, J. (2001). Physics, the Human Adventure: From Copernicus to Einstein and Beyond. Rutgers U. P., New Brunswick, N.J.

Hoyle, F. (1973). Nicolaus Copernicus: an essay on his life and work. Ist edition. Heinemann., London.

Kepler, J. (1609). Astronomia Nova.

King, A. (1993). From sage on the stage to guide on the side. College teaching, 4l(1):30-35. https://doi.org/10.1080/ 87567555.1993.9926781.

Kuhn, T. (1962). The structure of scientific revolutions. University of Chicago Press, Chicago, IL.

Leinhardt, G. (1988). Getting to know: Tracing students' mathematical knowledge from intuition to competence. Educational Psychologist, 23(2):119-144. https://doi.org/10. $1207 /$ s15326985ep2302_4.

Mach, E. (1872/1911). The history \& root of the principle of the conservation of energy. Open Court, Chicago, IL.

Mach, E. (1943/1895). On instruction in the classics \& the sciences. In Popular Scientific Lectures. Open Court. La Salle, Chicago.

Mansouri, F. A., Tanaka, K., and Buckley, M. J. (2009). Conflictinduced behavioural adjustment: a clue to the executive functions of the prefrontal cortex. Nature Reviews Neuroscience, 10(2):141-152. https://doi.org/10.1038/nrn2538.

Marion, J. and Thornton, S. (2020). Classical Dynamics of Particles and Systems. 3rd Edition. Harcourt Brace Jovanovich, New York, NY.

Marton, F. and Booth, S. (1997). Learning and Awareness. Lawrence Erlbaum, Mahwah, NJ.

Marton, F. and Pang, M. F. (2008). The idea of phenomenography and the pedagogy for conceptual change. In Vosniadou, S., editor, International handbook of research on conceptual change, pages 553-559. Routledge, London, UK.

Marton, F., Runesson, U., and Tsui, A. B. M. (2004). The space of learning. In Marton, F., Tsui, A. B. M., Chik, P. P., Ko, P., and Lo, M., editors, Classroom discourse and the space of learning, pages 43-62. Lawrence Erlbaum, Mahwah, NJ.

Matthews, M. R. (1989). A role for history and philosophy in science teaching. Interchange, 20(2):3-15. https://doi.org/ 
10.1007/BF01807043.

Monk, M. and Osborne, J. (1997). Placing the history and philosophy of science on the curriculum: A model for the development of pedagogy. Science education, 81(4):405424. https://doi.org/10.1002/(SICI) 1098-237X (199707)81: 4\%3C405: : AID-SCE3\%3E3.0.CO;2-G.

Murray, C. D. and Dermott, S. F. (1999). Solar System Dynamics. Cambridge University Press.

Orgill, M. (2012). Variation theory. In Seel, N. M., editor, Encyclopedia of the sciences of learning, page 3391-3393. Springer-Verlag Gmbm, Heidelberg, Germany.

Pang, M. F. and Marton, F. (2003). Beyond "lesson study": Comparing two ways of facilitating the grasp of some economic concepts. Instructional Science, 31(3):175-194. https:// doi.org/10.1023/A:1023280619632.

Piaget, J. (1970). Genetic Epistemology. Columbia University Press, New York, NY.

Piaget, J. (1977). The development of thought: Equilibration of cognitive structures. Viking, New York, NY.

Posner, G. J., Strike, K. A., Hewson, P. W., et al. (1982). Accommodation of a scientific conception: Toward a theory of conceptual change. Science education, 66(2):211-227. https : //doi.org/10.1002/sce.3730660207.

Rein, H. and Liu, S.-F. (2012). Rebound: an open-source multipurpose $n$-body code for collisional dynamics. Astronomy \& Astrophysics, 537:A128.

Rice, M. J. (1972). The case for the disciplines in the organization of social studies curricula for elementary and secondary schooling. paper presented at the colleges and university faculty association of the rational council for the social studies. Technical report, Boston, Massachussets.

Ryden, B. and Peterson, B. (2020). Foundations of Astrophysics. Cambridge University Press, Cambridge.

Saumure, K. and Given, L. (2008). Convenience sample. In Given, L., editor, The SAGE encyclopedia of qualitative research methods, pages 125-126. SAGE Publications, Thousand Oaks, CA.

Schwab, J. J. (1962). The teaching of science as inquiry. In Schwab, J. J. and Brandwein, P., editors, The teaching of science, pages 125-126. Harvard University Press, Cambridge, MA.

Setyadin, A. H., Suryana, T. G. S., Utari, S., Efendi, R., Liliawati, W., and Utama, J. A. (2020). Identifying k-10 students' learning difficulties on learning kepler's law using worksheet: Is it worth? In Journal of Physics: Conference Series, volume 1467, page 012051. IOP Publishing. https://doi.org/10. 1088/1742-6596/1467/1/012051.

Simon, M. A. (1995). Reconstructing mathematics pedagogy from a constructivist perspective. Journal for research in mathematics education, 26(2):114-145. https://doi.org/ 10.5951/jresematheduc.26.2.0114.

Waxer, M. and Morton, J. B. (2012). Cognitive conflict and learning. In Seel, N. M., editor, Encyclopedia of the Sciences of Learning, pages 585-587. Springer US, Boston, MA. https: //doi.org/10.1007/978-1-4419-1428-6_280.

Welch, W. W., Klopfer, L. E., Aikenhead, G. S., and Robinson, J. T. (1981). The role of inquiry in science education: Analysis and recommendations. Science Education, 65(1):3350. https://onlinelibrary.wiley.com/doi/abs/10.1002/sce. 3730650106.

Yu, K. C., Sahami, K., and Denn, G. (2010). Student ideas about kepler's laws and planetary orbital motions. Astronomy Education Review, 9(1). https://doi.org/10.3847/AER2009069.

Zazkis, R. and Chernoff, E. (2006). Cognitive conflict and its resolution via pivotal/bridging example. In Novotná, J., Moraová, H., Krátká, M., and Stehlíková, N., editors, Proceedings of the 30th Annual Conference of the International Group for the Psychology of Mathematics Education 5, pages 465-
472, Prague. PME. http://citeseerx.ist.psu.edu/viewdoc/ summary?doi=10.1.1.615.9518. 